\title{
Phonon-Assisted Exciton Transfer into Silicon Using Nanoemitters: The Role of Phonons and Temperature Effects in Förster Resonance Energy Transfer
}

\author{
Aydan Yeltik, ${ }^{\dagger, \ddagger}$ Burak Guzelturk, ${ }^{\dagger, \S, \ddagger}$ Pedro Ludwig Hernandez-Martinez, ${ }^{\dagger, \S}$ Alexander O. Govorov ${ }_{,}^{\perp}$ and \\ Hilmi Volkan Demir ${ }^{\dagger, \S, *}$ \\ ${ }^{\dagger}$ Department of Physics and Department of Electrical and Electronics Engineering, UNAM-Institute of Materials Science and Nanotechnology, Bilkent University, \\ Ankara 06800, Turkey, 'Luminous! Center of Excellence for Semiconductor Lighting and Displays, School of Electrical and Electronic Engineering and \\ School of Physical and Mathematical Sciences, Nanyang Technological University, Nanyang Avenue, Singapore 639798, Singapore, and ${ }^{\perp}$ Clippinger Research \\ Laboratories, Department of Physics and Astronomy, Ohio University, Athens, Ohio 45701, United States. ${ }^{\ddagger}$ A. Yeltik and B. Guzelturk contributed equally.
}

\begin{abstract}
We study phonon-assisted Förster resonance energy transfer (FRET) into an indirect band-gap semiconductor using nanoemitters. The unusual temperature dependence of this energy transfer, which is measured using the donor nanoemitters of quantum dot (QD) layers integrated on the acceptor monocrystalline bulk silicon as a model system, is predicted by a phonon-assisted exciton transfer model proposed here. The model includes the phonon-mediated optical properties of silicon, while considering the contribution from the multimonolayerequivalent QD film to the nonradiative energy transfer, which is derived with a $d^{-3}$ distance dependence. The FRET efficiencies are experimentally observed to decrease at cryogenic temperatures, which are well explained by the model considering the phonon depopulation in

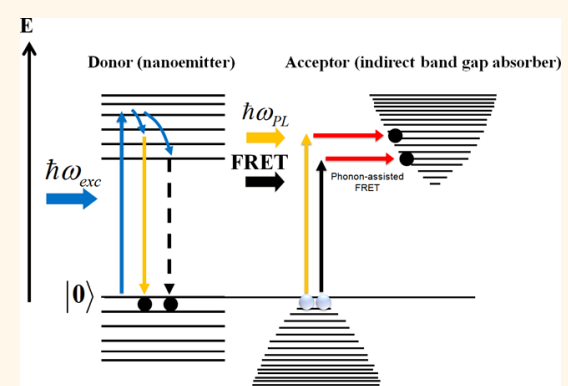

Phonon assistance in FRET the indirect band-gap acceptor together with the changes in the quantum yield of the donor. These understandings will be crucial for designing FRETenabled sensitization of silicon based high-efficiency excitonic systems using nanoemitters.
\end{abstract}

KEYWORDS: nonradiative energy transfer - NRET · Förster resonance energy transfer · FRET · phonons · temperature · indirect band-gap semiconductor $\cdot$ silicon $\cdot$ nanoemitters $\cdot$ quantum dots $\cdot$ exciton-exciton coupling

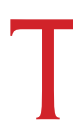
he phonon-assisted absorption of light in indirect band-gap semiconductors such as silicon (Si) is a fundamentally important optical process, which is essential to photovoltaic solar conversion. Optical transitions via phonon assistance in indirect band-gap silicon, which is the dominant material in photovoltaics today, ${ }^{1}$ have been extensively studied. The necessity of phonons for indirect interband transitions, however, imposes a significant constraint on the performance of the silicon-based solar cells. For increasing the probability of photon-phonon interaction and, in the end, enhancing optical absorption of silicon, there have been numerous efforts including light trapping, ${ }^{2-4}$ light field localization, 5,6 and those using external light sensitizers via transferring photon energy into silicon. ${ }^{7-10}$ For the latter purpose, colloidal quantum dots (QDs) have been proposed as good external sensitizer material systems owing to their favorably large absorption cross-section and easily tunable optical properties. To this end, QD sensitization on monocrystalline silicon has been previously demonstrated using radiative energy transfer (RET) between QDs and silicon. ${ }^{7,8}$ Although the sensitization of silicon through RET has been shown to lead to considerable improvement in the absorption properties of silicon-based devices, it is fundamentally restricted due to limited coupling of photons emitted by QDs into silicon. ${ }^{8}$ Alternatively, Förster resonance energy transfer (FRET), also known as nonradiative energy transfer (NRET) in general, which relies on near-field dipole-dipole interaction, ${ }^{11}$ is a promising method for the spectral sensitization of silicon via QDs. ${ }^{11-17}$ The advantage of these FRET-enabled sensitization systems is the ability of directly

\section{* Address correspondence to volkan@bilkent.edu.tr, hvdemir@ntu.edu.sg.}

Received for review March 9, 2013 and accepted November 25, 2013.

Published online November 25, 2013 10.1021/nn404627p

() 2013 American Chemical Society 
coupling the excitation energy in the strongly absorbing QDs to silicon while avoiding the otherwise necessary exciton recombination process in the sensitizer.

Previously, exciton transfer into silicon via FRET has been verified using time-resolved fluorescence (TRF) spectroscopy and time-resolved photocurrent measurements. ${ }^{11,15,16}$ However, this transfer was treated as the conventional FRET between a luminescent donor and a luminescent acceptor, although silicon is an indirect band-gap material and phonons are heavily involved in the optical transitions within silicon. It has been unknown to date whether there exists any fundamental difference in the underlying mechanism of the FRET into an indirect band-gap nonluminescent material that relies on phonon-mediated absorption.

In this work, we study the phonon-assisted FRET into indirect band-gap semiconductor silicon as a function of temperature to elucidate the underpinning physics behind the dynamics of this exciton transfer process using the donor nanoemitters integrated on the acceptor monocrystalline bulk silicon. Here we show that Förster-type NRET from nanoemitters into the silicon is a phonon-assisted process, like its optical counterpart RET. We propose and develop for the first time a phonon-assisted exciton transfer model that predicts the temperature-dependent dynamics of FRET in these QD-Si hybrid structures. Although FRET is an emissionless process, optical properties of the acceptor strongly modify the dynamics of FRET. The FRET efficiencies are experimentally observed to decrease at cryogenic temperatures, which are well explained by the model considering the phonon depopulation in the indirect band-gap acceptor together with the changes in the quantum yield (QY) of the donor. These understandings will be crucial for designing excitonic sensitization of silicon for high-efficiency light-harvesting systems.

\section{RESULTS AND DISCUSSION}

In this study, we design and fabricate hybrid nanostructures of a multimonolayer-equivalent film of core/ shell CdSe/ZnS QDs on single-crystal silicon. Figure 1a depicts the general structure of the working samples. Here, the bulk monocrystalline p-type silicon (100) substrate is utilized as the acceptor and the core/shell CdSe/ZnS QDs as the donor. The QDs were obtained from Evident Technologies and used without further purification ( $20 \mathrm{mg} / \mathrm{mL}$ in toluene). The peak emission wavelength of the QDs is $580 \mathrm{~nm}$ with a full-width at half-maximum (fwhm) of $35 \mathrm{~nm}$. The average radius of the QDs was obtained to be $2.22 \mathrm{~nm}$ from the ensemble high-resolution transmission electron microscopy (HR-TEM) measurements. The QDs are covered with hexadecylamine (HDA) ligands having a length of $\sim 2 \mathrm{~nm}$. The QDs were spin-coated over the $\mathrm{Al}_{2} \mathrm{O}_{3} /$ $\mathrm{SiO}_{2} / \mathrm{Si}$ structures, resulting in the formation of an approximately 10-monolayer-equivalent QD film as measured by optical ellipsometry (V-Vase J.A.Woolam). The QD film is uniformly distributed, as verified by scanning electron microscopy (SEM) and atomic force microscopy (AFM), as shown in Figure $1 \mathrm{~b}$ and c. As presented by the SEM image in Figure $1 b$, the top QD layer is highly packed without any aggregate formation, indicating the high film quality. The surface coverage fraction of the QDs reaches $\sim 65 \%$ as obtained by analysis of the SEM image in Figure 1b. Furthermore, AFM images prove that the surface uniformity is quite high with only one QD (with ligand) thick voids on the sample (see Figure 1c and height profile). Overall rms roughness is found to be $\sim 2 \mathrm{~nm}$, which is much smaller than the size of the QDs. Therefore, the QD film deposited upon silicon is high quality; thus, the sample can be considered as 10-monolayer-equivalent, although it is not prepared by layer-by-layer deposition. From the ensemble TEM images, the center-to-center distance between two adjacent QDs was estimated to be $6.48 \mathrm{~nm}$, including the interpenetrating ligands. QYs of the 10-monolayer-equivalent QD film on sapphire and the diluted QD solution were measured as $25 \%$ and $29 \%$, respectively, using an integrating sphere (Horiba Jobin Yvon PL spectrometer equipped with a QY measurement setup). The employed single-crystal silicon is $0.5 \mathrm{~mm}$ thick and possesses approximately $1.65 \mathrm{~nm}$ thick native oxide on the top, as verified by the optical ellipsometry measurements. To incorporate a controlled separation between the QDs and silicon, we deposited $\mathrm{Al}_{2} \mathrm{O}_{3}$ film on precleaned silicon substrates having the native oxide by using the atomic layer deposition (ALD) technique, which is one of the most suitable methods to deposit atomically thin layers conformably and controllably. The film thicknesses of the $\mathrm{Al}_{2} \mathrm{O}_{3}$ layer were carefully set to $1.0,2.0$, and $4.0 \mathrm{~nm}$ and verified using ellipsometry measurements. In addition, the same amount of QD-coated sapphire $\left(\mathrm{Al}_{2} \mathrm{O}_{3}\right)$ substrate was utilized as a reference sample, where no FRET exists. The inset in Figure 1b shows optical absorption and photoluminescence (PL) spectra of the QDs when pumped at $350 \mathrm{~nm}$.

While performing the time-resolved fluorescence spectroscopy, the peak emission wavelength of the QDs is monitored via the time-correlated single photon counting technique. Measurements were performed for different $\mathrm{Al}_{2} \mathrm{O}_{3}$ spacer thicknesses at varying temperatures between 22 and $290 \mathrm{~K}$ using a closed-cycle He cryostat. The optical absorption spectrum of silicon is also depicted (in log scale in the inset of Figure $1 \mathrm{~b}$ ) to illustrate the spectral overlap between the silicon's absorption and the QDs' emission.

Figure 2 depicts the exemplary room-temperature fluorescence decay curves of the 10-monolayer-equivalent film of QDs deposited on the $\mathrm{Al}_{2} \mathrm{O}_{3} / \mathrm{SiO}_{2} / \mathrm{Si}$ layered samples for varying $\mathrm{Al}_{2} \mathrm{O}_{3}$ spacer thicknesses $(X=0.0$ to $4.0 \mathrm{~nm})$ and on the sapphire $(X=$ inf). The corresponding fluorescence lifetimes were also recorded 

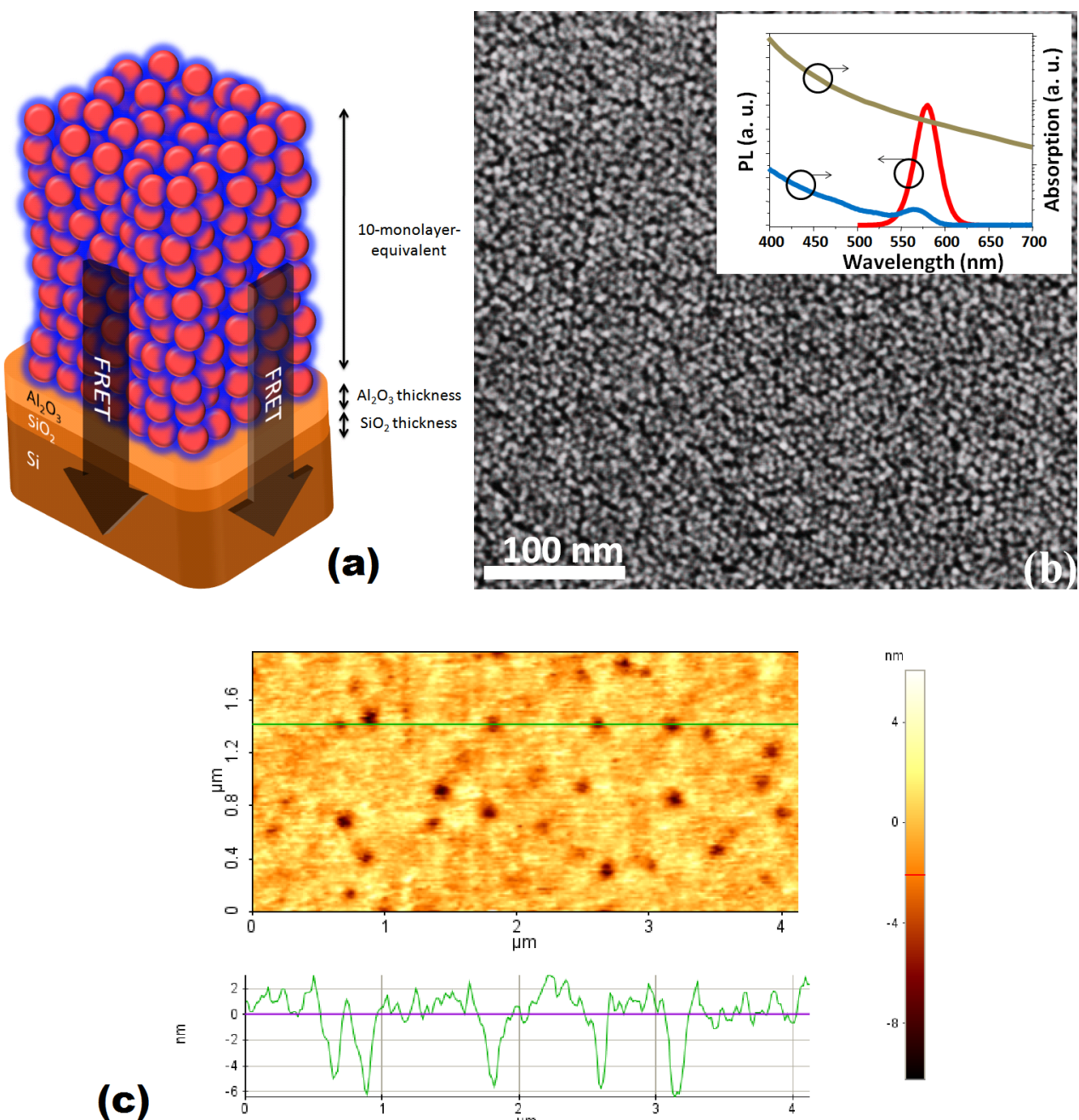

(c)

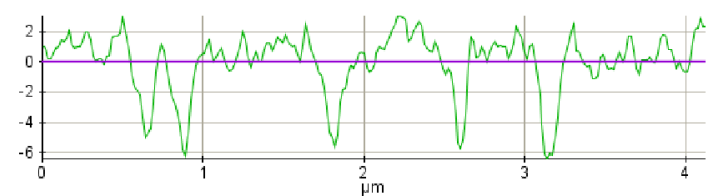

Figure 1. (a) Schematic of the hybrid nanostructure of multimonolayer QDs and silicon separated by controlled $\mathrm{Al}_{2} \mathrm{O}_{3}$ separation thickness. Here, the native $\mathrm{SiO}_{2}$ thickness is $1.65 \mathrm{~nm}$. The thickness of $\mathrm{Al}_{2} \mathrm{O}_{3}$ film is varied from 0.0 to $4.0 \mathrm{~nm}$. The QDs of the same monolayer are assumed to have the same exciton transfer contribution to bulk silicon. (b) SEM image of the QDs furnished on the $\mathrm{Al}_{2} \mathrm{O}_{3} / \mathrm{SiO}_{2} / \mathrm{Si}$ structures. Inset shows optical absorption and PL spectra of the QDs (black and green curves, respectively) and absorption spectrum of silicon (red curve). (c) Atomic force microscopy image of the 10-monolayer-equivalent QD film on top of silicon with the height profile of the line shown inside the AFM image.
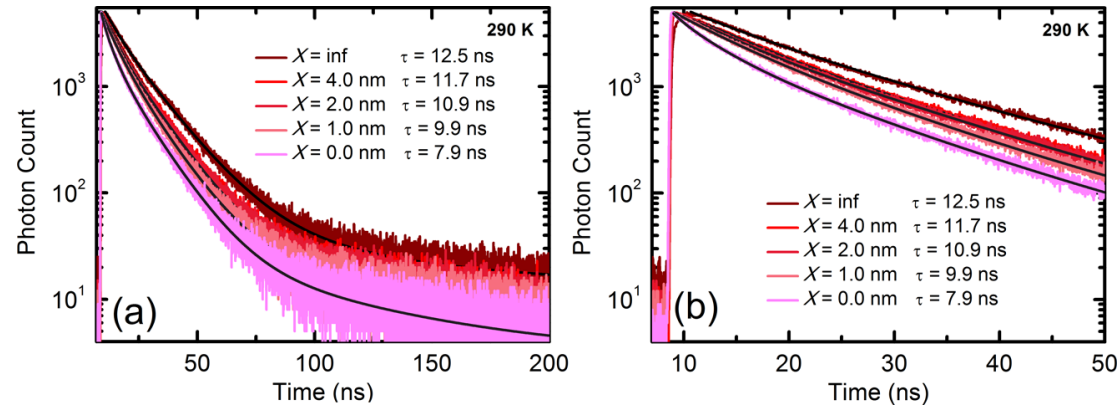

Figure 2. Experimental fluorescence decays of a 10-monolayer-equivalent QD film on a silicon substrate with a 0.0, 1.0, 2.0, and $4.0 \mathrm{~nm}$ of $\mathrm{Al}_{2} \mathrm{O}_{3}$ separation layer and on sapphire as reference sample: (a) over a time window of $200 \mathrm{~ns}$ and (b) zooming in the first $50 \mathrm{~ns}$. The multiexponential fits are shown inside the decay curves with the average lifetimes reported.

as shown in Figure 2. The decay curves are fitted with multiexponential lifetimes, producing reduced $\chi^{2}$ close to 1 , and the exciton lifetimes are calculated via amplitude averaging, since there is only one type of fluorophore (i.e., the QDs) in the system. Owing to the increased dipole-dipole coupling at short donor-acceptor 


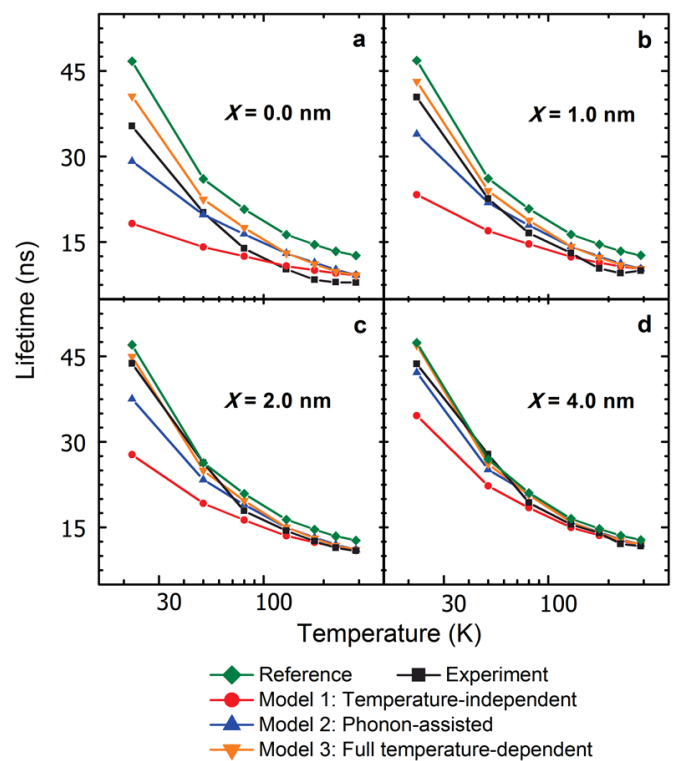

Figure 3. Temperature dependence of the fluorescence lifetime of the QDs integrated on top of a (a) $0.0 \mathrm{~nm}$, (b) $1.0 \mathrm{~nm}$, (c) $2.0 \mathrm{~nm}$, and (d) $4.0 \mathrm{~nm}$ thick $\mathrm{Al}_{2} \mathrm{O}_{3}$ layer on $\mathrm{SiO}_{2} /$ Si. Black squares are amplitude-averaged experiment lifetimes. Green diamonds are the lifetimes of the QDs on sapphire as the reference sample corrected for the refractive index difference with silicon. Red circles are the calculated lifetimes of the QDs using the "temperatureindependent" energy transfer model. Blue up-triangles are the calculated lifetimes of the QDs using the energy transfer model, which considers the temperature-dependent complex dielectric function of silicon, thus being a "phonon-assisted" model. Orange down-triangles are the calculated lifetimes of the QDs using the "phonon-assisted" energy transfer model with the additional inclusion of the temperature-dependent $\mathrm{QY}$ of the donor QDs; thus, it is called a "full temperature-dependent" model.

separations, as the separation thickness is reduced from $4.0 \mathrm{~nm}$ to $0.0 \mathrm{~nm}$, the lifetimes of the QDs decreased, indicating that FRET becomes stronger at shorter separation distances (see Figure 2). Moreover, we checked the interdot FRET, which could be a dominant mechanism for the thick QD films. In our case, interdot FRET is found to be weak, since fluorescence lifetimes of the blue- and red-tail emission do not differ much (up to 2.5-fold) as compared to the lifetime at the peak emission wavelength. This is related to the fact that self-spectral overlap is not strong enough to realize dominant interdot FRET taking into account the dot-to-dot separation due to the shell and ligand length (see Supporting Information for more details). However, as we observed from our trials with either small sized core or thin shell QDs, where interdot FRET tends to become dominant, the analysis of the FRET into silicon is not straightforward.

In this study, we employ five different samples: 10-monolayer-equivalent QDs on top of either sapphire (reference) or $0.0,1.0,2.0$, or $4.0 \mathrm{~nm} \mathrm{Al}_{2} \mathrm{O}_{3}$ separated $\mathrm{SiO}_{2} / \mathrm{Si}$ substrates. Temperature-dependent amplitudeaveraged fluorescence lifetimes of the QDs grafted on these five different substrates are presented in Figure $3 a-d$ for the cases of $0.0,1.0,2.0$, and $4.0 \mathrm{~nm}$ thick $\mathrm{Al}_{2} \mathrm{O}_{3}$ separation, respectively. Green diamonds are the experimental reference lifetimes (QDs on sapphire: no FRET), which are corrected for the refractive index difference between sapphire and silicon, causing radiative energy transfer, the reason for this correction, which will be explained in the next part. Black squares are the experimental lifetimes of the QDs when placed on $\mathrm{SiO}_{2} / \mathrm{Si}$ with a respective separation thickness. As a general trend, the lifetimes of the QDs are observed to increase with decreasing temperature, which is in agreement with previous reports. ${ }^{18}$

As stated earlier, fluorescence lifetimes of the QDs on sapphire (reference sample, green diamonds in Figure 3) have to be corrected due to refractive index difference between sapphire and silicon to correctly account for the reference lifetimes. This effect is summarized in terms of rate equations below:

$$
\begin{gathered}
\gamma_{\mathrm{Al}_{2} \mathrm{O}_{3}}=\gamma_{\mathrm{r}}+\gamma_{\mathrm{nr}}+\gamma_{\mathrm{Al}_{2} \mathrm{O}_{3}, \mathrm{RET}} \\
\gamma_{\mathrm{Si}}=\gamma_{\mathrm{r}}+\gamma_{\mathrm{nr}}+\gamma_{\mathrm{Si}, \mathrm{RET}}+\gamma_{\mathrm{FRET}}
\end{gathered}
$$

$\gamma_{\mathrm{r}}$ and $\gamma_{\mathrm{nr}}$ are the radiative and nonradiative decay rates of the QDs in a vacuum. $\gamma_{\mathrm{Al}_{2} \mathrm{O}_{3}, \mathrm{RET}}$ and $\gamma_{\mathrm{Si}, \mathrm{RET}}$ are the radiative energy transfer terms arising due to the difference of the refractive index of the substrate and the vacuum, which alters the radiative decay rate of the QDs. RET terms are different for the case of sapphire and silicon due to the difference of the refractive indices; therefore we calculate a correction factor for the reference sample to account for the difference in refractive indices. For this correction, the model developed by Chance, Prock, and Silbey, as introduced in ref 19 , is adapted while taking the radiative decay of a randomly oriented dipole into account in the presence of reflected electric fields due to the inhomogeneous environment. The changes of QD lifetimes from on sapphire to on hypothetical-silicon, having no absorption component in the dielectric function, were calculated. ${ }^{17,20}$ The radiative lifetime of a QD placed on hypothetical-silicon is decreased by $\sim 2.1$ times as compared to the one placed on sapphire. In our case, since a 10-monolayer-equivalent QD film has been employed as the sample, and the QY of the QD film was not unity (it is measured as $25 \%$ ), the correction factor boils down to a smaller value of $\sim 1.1$ (see the Supporting Information for detailed calculation of the correction factors, section B; eqs S24, S25, and S26; and section $\mathrm{E})$. After the correction, RET terms in eqs 1 and 2 become equal so that the extra change of the decay rate on silicon could be attributed to the term $\gamma_{\text {FRET, }}$ which is the nonradiative energy transfer of the excitons in the QDs to silicon via near-field dipole-dipole coupling.

To develop a deeper understanding of the FRET between the QDs and silicon, we propose a physical model for the energy transfer across the studied hybrid 
QD-Si nanostructure. Figure 4 illustrates the dipoledipole Coulomb interaction between the donor QD and the acceptor silicon. An absorbed photon in a QD photogenerates a bound electron-hole pair (exciton), which rapidly relaxes to the first excited state through higher order processes. These excitons can subsequently either recombine through a radiative or nonradiative means inside the $\mathrm{QD}$ or be transferred to silicon via Coulomb interaction between dipoles in the donor-acceptor pair provided that the separation distance between the donor and the acceptor is on the order of a few nanometers. Although this type of energy transfer has been well known and studied as Förster resonance energy transfer, it has not been fully understood when an indirect band-gap acceptor is involved. Previously, Stavola et al. discussed the effects of donor-acceptor distance to FRET, while having an indirect band-gap acceptor. ${ }^{12}$ There, they have defined proximity effects, which were calculated to be dominant for the donor-acceptor separations on the order of a few lattice constants of the acceptor crystal. Here, the smallest donor-acceptor separation is $5.9 \mathrm{~nm}$ (distance from the center of the closest QD layer to the surface of silicon for the case of no alumina separation), which is much larger than the silicon's lattice constant $(\sim 0.54 \mathrm{~nm})$. Therefore, the transitional interactions can be assumed to occur mainly in the optical near-field region around $k \approx 0.1^{13,21}$ To date, such phonon-mediated FRET dynamics have not been studied in terms of physical models for this kind of unique donor-acceptor material systems.

To estimate the exciton transfer from the QD to bulk silicon, the well-known Fermi's golden rule is used. After using the fluctuation dissipation theorem ${ }^{22}$ and algebraic manipulation (see the Supporting Information for derivations), the resulting FRET rate can be derived in a form convenient for numeric calculations. ${ }^{23,24}$ To understand the origins of the temperature dependence in transfer, we will examine three forms of the equation for the FRET rate:

$$
\gamma_{\text {NRET, Palik }}\left(d, \omega_{\text {exc }}\right)=F(d) \cdot \operatorname{Im}\left[\varepsilon_{\text {Si, Palik }}\left(\omega_{\text {exc }}\right)\right]
$$

$\gamma_{\mathrm{NRET}, T \text {-dependence }}\left(d, \omega_{\mathrm{exc}}, T\right)=F(d, T) \cdot \operatorname{Im}\left[\varepsilon_{\mathrm{Si}}\left(\omega_{\mathrm{exc}}, T\right)\right]$

$\gamma_{\mathrm{NRET}, \text { full }}\left(d, \omega_{\mathrm{exc},}, T\right)=\left(\frac{Y_{\mathrm{QD}}(T) \gamma_{0}^{D}(T)}{Y_{\mathrm{QD}}\left(T_{\mathrm{Room}}\right) \gamma_{0}^{D}\left(T_{\mathrm{Room}}\right)}\right) F(d, T) \cdot \operatorname{Im}\left[\varepsilon_{\mathrm{Si}}\left(\omega_{\mathrm{exc}}, T\right)\right]$

$$
F(d, T)=\frac{2}{\hbar}\left(\frac{1}{12}\right)\left(\frac{\mathrm{ed}_{\mathrm{exc}}}{\varepsilon_{\mathrm{eff}}}\right)^{2} \frac{1}{d^{3}}\left|\frac{2 \varepsilon_{0}}{\varepsilon_{\mathrm{Si}}(T)+\varepsilon_{0}}\right|^{2}
$$

where ed $\mathrm{exc}_{\mathrm{exc}}$ and $\omega_{\text {exc }}$ are the exciton dipole moment and angular frequency, respectively; $d$ is the QDsilicon separation distance, $\varepsilon_{\text {eff }}=\left(2 \varepsilon_{0}+\varepsilon_{\mathrm{QD}}\right) / 3$ is the effective dielectric constant, $\varepsilon_{\mathrm{QD}}$ is the QD dielectric

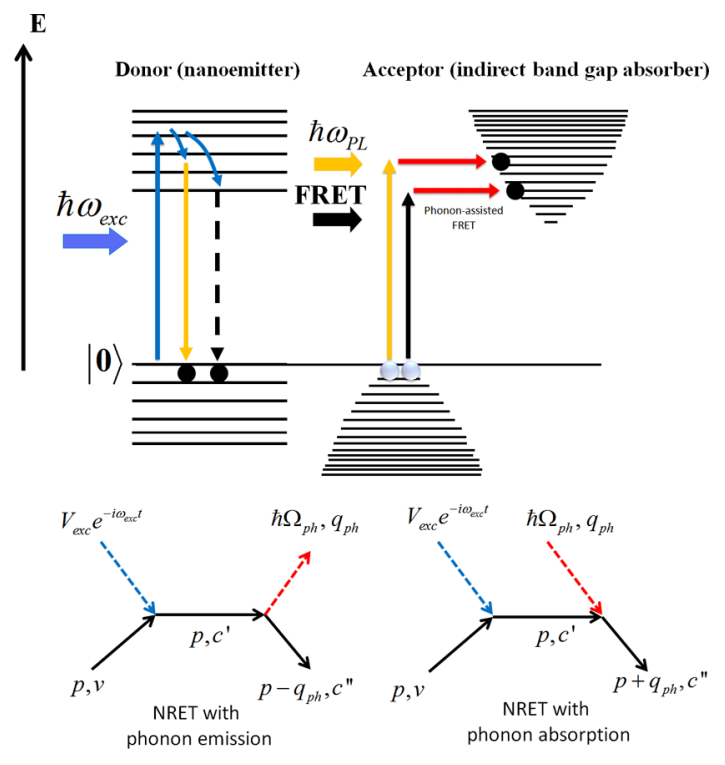

Figure 4. (a) Energy diagram illustrating the exciton transfer from the donor QD to the acceptor silicon due to the Coulomb interaction between the donor-acceptor pair. The phonon-assisted process is shown as the lateral arrows to make up for the momentum mismatch in silicon. (b) Some of the Feynman diagrams for the phonon-assisted processes important for transfer of energy from a QD to an indirect-band semiconductor. These diagrams include phonon-emission processes in the conduction band.

constant, $\varepsilon_{0}$ is the medium dielectric constant, and $\varepsilon_{\mathrm{Si}}(\omega, T)$ is the silicon dielectric function. ${ }^{25}$ In eq 3 , we assumed $\operatorname{Re}\left[\varepsilon_{\mathrm{Si}}(\omega, T)\right]=\varepsilon_{\mathrm{Si}} \gg \operatorname{Im}\left[\varepsilon_{\mathrm{Si}}(\omega, T)\right]$, which is a very good approximation for the considered exciton energy $\left(\lambda_{\mathrm{exc}} \approx 580 \mathrm{~nm}, \hbar \omega_{\mathrm{exc}} \approx 2.1 \mathrm{eV}\right)$. The function $F(d)$ in eq 3 involves the optical dipole moment of QD, which is taken as $d_{\text {exc }}=0.4 \mathrm{~nm}$. It is estimated from the QD exciton lifetime of the reference sample. This exciton dipole moment was estimated from the exciton lifetime and the QY of QDs in the reference sample. Here, we assume that the QDs on the quasi-monolayer have the same exciton transfer contribution to bulk silicon, such that the average exciton transfer can be described by the energy transfer of a single $Q D$ in a given monolayer to bulk silicon. In this derived analytical model, it is worth noting that the distance dependence of the FRET rate is proportional to $d^{-3}$, which is because the acceptor is bulk. This distance dependence is quite different from FRET in the dye-dye ${ }^{11}$ and QD-QD ${ }^{26,27}$ systems, where a FRET rate for a donor-acceptor pair is $\sim d^{-6}$. Here one fixed set of material parameters is employed for all samples, and no fetch factor is employed (see Supporting Information for the parameters).

Including the FRET process, the lifetimes of the QDs on the $\mathrm{Al}_{2} \mathrm{O}_{3} / \mathrm{SiO}_{2} / \mathrm{Si}$ substrates can be calculated in the following way:

$$
\gamma(d, \omega)=\left\langle\gamma_{0, \text { ref }}^{*}(d, \omega)\right\rangle+\frac{1}{N} \sum_{i=1}^{N} \gamma_{i, \mathrm{NRET}}\left(d_{i}, \omega\right)
$$


Here, $\gamma(d, \omega)$ is the decay rate of the QDs on the $\mathrm{Al}_{2} \mathrm{O}_{3} /$ $\mathrm{SiO}_{2} / \mathrm{Si}$ layer, $\left\langle\gamma_{0, \text { ref }}^{*}(d, \omega)\right\rangle$ is the decay rate of QDs corrected for the refractive index difference as obtained from the reference sample (see eq S26 in the Supporting Information for more details), $N$ is the total number of monolayers, and $\gamma_{i, \mathrm{NRET}}\left(d_{i}, \omega\right)$ is the FRET rate for each corresponding layer. The contribution coming from each different QD layer was taken into account by considering the respective separation of the given monolayer to the bulk silicon. Practically, it is assumed that after the fifth QD layer there is no considerable FRET into silicon; thus the entire FRET is caused by the first five QD layers, and it is averaged over the total decay of the 10 QD layers (see eq 4). Interdot FRET is ruled out here, since the same QD film (10 monolayerequivalent $\mathrm{QDs}$ ) on sapphire is utilized as the reference sample.

Now, we briefly show the derivation for the microscopic mechanisms of phonon-assisted FRET, which are hidden in the key function $\operatorname{Im}\left[\varepsilon_{\mathrm{Si}}\left(\omega_{\text {exc }} T\right)\right]$ in eq 3 . Below we will see that the theory based on the experimental dielectric constants taken from refs 25 and 28 provides us with an overall good description of the temperature and distance trends for the FRET rates. The imaginary part of the dielectric constant and the related absorption in silicon at the peak QD emission wavelength are due to the phonon-assisted processes ${ }^{29}$ shown in Figure 4. To illustrate the process of phononassisted FRET to an indirect semiconductor, we now consider transfer of a $\mathrm{QD}$ exciton energy to a small volume, $\Delta V$, at the position $R_{0}$ within the Si half-space. An exciton in a QD can be viewed as an oscillating dipole creating a time-varying electric field $E_{\text {exc }}$. The rate of energy dissipation due to the field of an oscillating dipole in the volume $\Delta V$ is

$$
\Delta Q=\Delta V\left|E_{\mathrm{exc}}\right|^{2} \frac{\omega_{\mathrm{exc}}}{2 \pi} \operatorname{Im}\left[\varepsilon_{\mathrm{si}}\left(\omega_{\mathrm{exc}}, T\right)\right]
$$

The absorption rate can also be calculated by the Feynman diagrams (two of them are shown in Figure $4 b$ ) in combination with Fermi's golden rule:

$$
\begin{aligned}
& \Delta Q=\Delta Q_{+}+\Delta Q_{-} \\
& \Delta Q_{ \pm}=\hbar \omega_{\mathrm{exc}} \frac{2 \pi}{\hbar}\left(\sum_{f=\left\{p, p^{\prime}\right\}}\left|\sum_{i} \frac{\left\langle f\left|\hat{V}_{\mathrm{e}-\text { phonon }}\right| i\right\rangle\left\langle i\left|\hat{V}_{\mathrm{exc}}\right| 0\right\rangle}{E_{0}+\hbar \omega_{\mathrm{exc}}-E_{i}}\right|^{2}\right. \\
& \left.\times \delta\left(\hbar \omega_{\mathrm{exc}}+\varepsilon_{p, v}-\varepsilon_{p^{\prime}, c^{\prime}} \pm \hbar \Omega_{q_{\mathrm{ph}}}\right)\right)
\end{aligned}
$$

In this equation, $\hat{V}_{\text {exc }}$ is the amplitude of the timeoscillating potential of the exciton and $\hat{V}_{\mathrm{e}-\text { phonon }}$ is the electron-phonon interaction in $\mathrm{Si} . E_{0}$ and $E_{i}$ are the energies of the initial and intermediate states, respectively, whereas $\varepsilon_{p, v(c)}$ denotes the energies of single electrons in the valence and conduction bands. The indices $p$ and $p^{\prime}$ describe the momenta of single electrons in the initial and final states. The functions $\Delta Q_{-}$and $\Delta Q_{+}$give the absorption processes with emission and absorption of phonons, respectively.
Correspondingly, the final states in eq 6 can be of two types:

$$
\left|p^{\prime}, c ; n_{q_{\mathrm{ph}}}-1\right\rangle \text { and }\left|p^{\prime}, c ; n_{q_{\mathrm{ph}}}+1\right\rangle
$$

These final states appear as a result of phonon-absorption and phonon-emission interband processes (Figure 4b). Here, the state $\left|n_{q_{\mathrm{ph}}}\right\rangle$ denotes an initial phonon state with an occupation number $n_{q_{\mathrm{ph}}}$, where $q_{\mathrm{ph}}$ is a phonon momentum. Equation 6 has very characteristic temperature- and exciton energy-dependencies. The phonon-emission term in $\hat{V}_{\mathrm{e}-\text { phonon }}$ will produce the standard factor $\left(n_{q_{\mathrm{ph}}}+1\right)^{1 / 2}$ inside the sum, and after taking the square it will become $n_{q_{\mathrm{ph}}}+1$. In the same way, the phonon-annihilation term in $\hat{V}_{\mathrm{e}-\text { phonon }}$ will give the factor $n_{q_{\mathrm{ph}}}$. The occupation numbers are, of course, given, by the Bose-Einstein function,

$$
n_{q_{\mathrm{ph}}}=n_{\mathrm{B}}\left(\omega_{\mathrm{ph}}\right)=\frac{1}{\mathrm{e}^{\hbar \omega_{\mathrm{ph}} / k_{\mathrm{B}} T}-1}
$$

The effective density of states for the indirect interband phonon-assisted transitions is well known and, near the band gap, has the form $\left(\hbar \omega-E_{\text {gap }}\right)^{2}$, where $E_{\text {gap }}$ is an indirect band gap of a semiconductor. ${ }^{29}$ These considerations allow us to write the dissipation and the imaginary part of the dielectric constant in the following way adopted from ref 28 :

$$
\begin{gathered}
\operatorname{Im}\left[\varepsilon_{\mathrm{Si}}\left(\omega_{\mathrm{exc}}, T\right)\right]=\frac{n_{\mathrm{Si}}^{0} c_{0}}{\omega_{\mathrm{exc}}} \alpha\left(\omega_{\mathrm{exc}}, T\right)=\left(\frac{n_{\mathrm{Si}}^{0} c_{0}}{\omega_{\mathrm{exc}}}\right) \\
\times \quad A_{j} C_{i} n_{\mathrm{B}}\left(\Omega_{\mathrm{ph}, i}, T\right) \\
i=1,2 \text { (phonons) } \\
j=1,2(\text { band-gaps in Si) } \\
\times\left[\hbar \omega-E_{g, j}(T)+\hbar \Omega_{\mathrm{ph}, i}\right]^{2}+A_{j} C_{i} \times\left[n_{\mathrm{B}}\left(\Omega_{\mathrm{ph}, i}, T\right)+1\right] \\
\times\left[\hbar \omega-E_{g, j}(T)-\hbar \Omega_{\mathrm{ph}, i}\right]^{2}
\end{gathered}
$$

where the empirical coefficients obtained from experiments ${ }^{28}$ are given in the Supporting Information in Section E. Equation 9 involves two types of optical phonons and two indirect band gaps typical for the silicon crystal and provides a rather realistic approximation for silicon. Importantly, two sources of temperature dependence can be identified from eq 9: the phonon populations $\left(n_{\mathrm{B}}\left(\Omega_{\mathrm{ph}, i}, T\right)\right)$ and the temperaturedependent band gaps. Both sources are important for $\mathrm{Si}$. The above consideration has shown the microscopic origin of the FRET process in the hybrid QD-Si system, in which phonon-assisted processes are crucial for energy transfer. This is in striking contrast to the systems with direct band-gap crystals, such as CdSe or CdTe systems, ${ }^{30}$ for example.

Here, using eqs 3, 4, and 9 the experimental data are contrasted at various temperatures with three different theoretical approaches: "temperature-independent", "phonon-assisted", and "full temperature-dependent".

1. Palik's Dielectric Function at $T=I_{\text {room }}$ : Model 1, Temperature Independent. Using the temperature-independent function $\gamma_{\text {NRET,Palik }}\left(\omega_{\text {exc }}\right)$ and eq $3 a$, we obtain the rates 
that have clearly a weaker $T$-dependence compared to the experimental data (Figure 3). The temperature dependence in this case comes from the intrinsic QD lifetime $\tau_{0}(T)$.

2. Temperature-Dependent Dielectric Function from Ref 28: Model 2, Phonon-Assisted FRET. To improve our model and include temperature dependence into the FRET rate, we now include the temperature-dependent processes in $\mathrm{Si}$ and use eq $3 \mathrm{~b}$ with the dielectric function from ref 28 . For different temperatures, a temperatureindependent refractive index is taken as 4 at the wavelengths of interest according to Bucher et $\mathrm{al}^{28}$ Results are given again in Figure 3. The insertion of the phonon-assisted absorption of silicon improved the theoretical prediction considerably, as shown in Figure 3 by blue up-triangles. The low-temperature agreement is much better compared to the bare energy transfer model, which does not involve any temperature-dependent process. This means that phonon-assisted properties of silicon as the acceptor in our energy-harvesting system directly affect the energy transfer rates. Generally, it is not common in the context of FRET being a phonon-assisted process, since most of the time the excitation energy is resonantly transferred to the state in the acceptor without absorption or emission of any phonons. However, it is well accepted that the radiative transitions in indirect bandgap materials involve phonons for the transitions to take place. In the energy transfer process from QDs to bulk silicon, similar to optical transitions, this suggests that phonons are required to assist the energy transfer. As a result, this type of energy transfer cannot be called merely Förster-type, since its resonance condition can be relaxed due to involvement of the phonons. Furthermore, the effects of different types of phonons on this energy transfer are different. Especially, acoustic phonons are considered responsible for the optical transitions at low temperatures due to their lower activation energies. At cryogenic temperatures, however, there are fewer optical phonons as compared to the acoustic ones, since the optical phonons have much higher activation energy. In addition, considering the optical absorption for silicon, which includes phonon absorption and emission effects in addition to direct transitions with no phonon assistance, the phonon emission is observed to be a more dominating process at low temperatures.

3. Temperature-Dependent QY of the Donor QDs: “Model 3, Full Temperature-Dependent Approach. Despite the reasonable agreement between the results of the phononincluded model and the experimental data, there remains some discrepancy especially at the cryogenic temperatures below $100 \mathrm{~K}$. We attribute it to the QD's temperature-dependent optical properties, i.e., QY. We measured and calculated the relative change of the temperature-dependent QY for the QDs and observed that, as the sample temperature is gradually decreased,

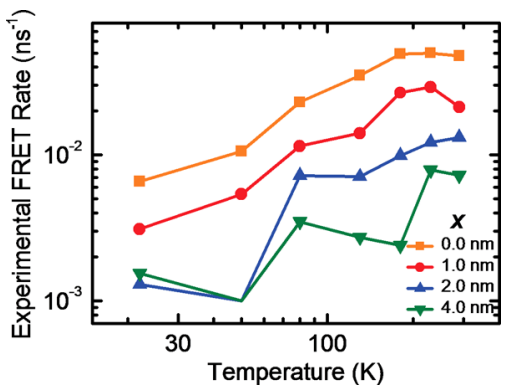

Figure 5. Experimental FRET rates as a function of temperature. Orange squares correspond to the FRET without $\mathrm{Al}_{2} \mathrm{O}_{3}$. Red circles correspond to the FRET for a $1.0 \mathrm{~nm} \mathrm{Al} \mathrm{O}_{3}$ thickness. Blue up-triangles correspond to the FRET for a $2.0 \mathrm{~nm} \mathrm{Al} \mathrm{O}_{3}$ thickness. Green down-triangles correspond to the FRET for a $4.0 \mathrm{~nm} \mathrm{Al} \mathrm{O}_{3}$ thickness. The FRET rates in this graph were obtained taking the modified reference rates into account.

the QY increases until $150 \mathrm{~K}$, peaking at this temperature, and then decreases toward lower temperatures (Figure S2). ${ }^{31}$ Our energy transfer model intrinsically includes the theoretical radiative decay rate of the donor QDs at room temperature. By further including the QY changes as a function of temperature in the model, we therefore modify the radiative decay rate of the QDs as a function of temperature in accordance with the experimentally measured total decay rates and the QYs. Using these temperature-dependent QYs and the ratio between the transfer rates of the QDs on the reference sample, we obtain the final modified model, which includes both temperature-dependent effects of the QDs and silicon as given in eq 3c. The final model results in an improved match between the theory and experiment especially at the lowest temperature cases, as shown in Figure 3. The "full temperature-dependent approach" reproduces qualitatively all experimentally observed trends for the FRET rate as a function of separation distance and temperature with a slight improvement over the "phonon-assisted" model, compared to the improvement of the "phonon-assisted" model introduced over the "temperature-independent" analytical one. All in all, the energy transfer model including the phonon-assisted processes in the absorption of silicon with the help of temperature-dependent optical properties in the QDs proved its effectiveness to assess temperature-dependent fluorescence lifetimes of the donor QDs better and showed reasonable agreement with the experimental measurements.

Experimental FRET rates are summarized in Figure 5. These rates were calculated as

$$
\gamma_{\mathrm{NRET}, \text { tot }}(T)=\gamma_{\mathrm{QD}, \mathrm{Si}}-\gamma_{\mathrm{QD}, 0}
$$

where $\gamma_{\mathrm{QD}, \mathrm{Si}}$ is the decay rate for QDs on Si and $\gamma_{\mathrm{QD}, 0}$ is the intrinsic decay rate of the excitons corrected due to the modified radiation probability on silicon. Theoretically, the FRET rates are obtained from 


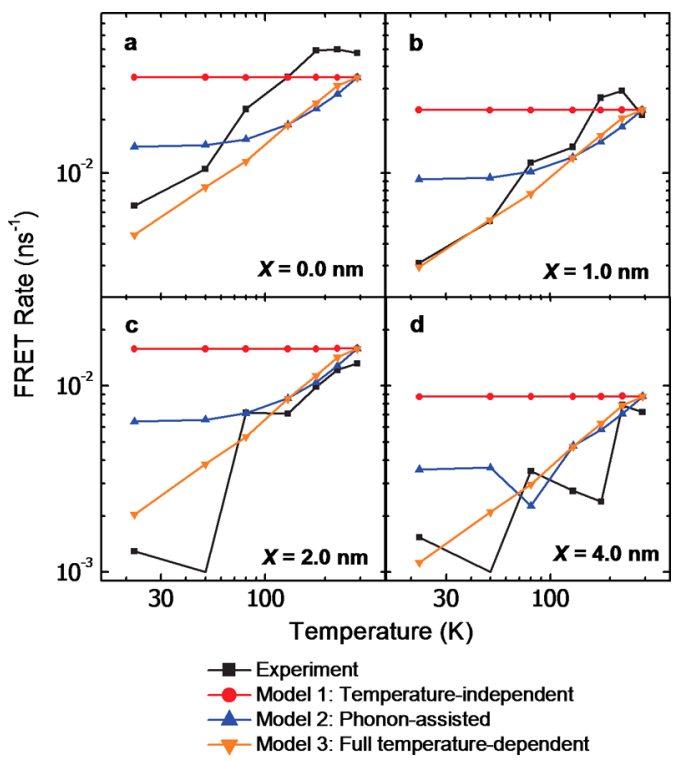

Figure 6. Energy transfer rates as a function of temperature for each case: $\mathrm{Al}_{2} \mathrm{O}_{3}$ thickness of $0.0,1.0,2.0$, and $4.0 \mathrm{~nm}$. Black squares correspond to the experimental data. Red circles represent the FRET without phonon assistance, blue up-triangles include phonon assistance, and orange downtriangles consider both the phonon assistance and temperature-dependent QY change of the QDs.

$\gamma_{\text {NRET, tot }}(T)=\frac{1}{N} \sum_{i=1}^{N} \gamma_{i, \text { NRET }}\left(d_{i}, \omega\right)=f_{d}\left(X_{\mathrm{Al}_{2} \mathrm{O}_{3}}\right) F_{T}\left(T, \omega_{\text {exc }}\right)$

We can see from the above equation that the FRET rate can be conveniently expressed as a product of two functions, $f_{d}\left(X_{\mathrm{Al}_{2} \mathrm{O}_{3}}\right)$ and $F_{T}\left(T, \omega_{\text {exc }}\right)$, where $X_{\mathrm{Al}_{2} \mathrm{O}_{3}}$ is the thickness of the separation layer. Figure 5 shows the initial experimental rates, whereas Figure 6 displays the comparison between the experimental and theoretical data. In Figure 5, we see clearly the trend; the FRET rates are much higher for small $\mathrm{Al}_{2} \mathrm{O}_{3}$ separations. This is expected, since the Coulomb-induced energy transfer process rapidly decays with increasing distance. Important results on the temperature dependence of FRET are shown in Figure 6. We see overall good agreement between the "full temperature-dependent" model and the experiment. After including both the temperature-dependent dielectric function of acceptor silicon and QY of the donor QDs, the theoretical model reasonably reproduces the experimental data. We note that the effect of the temperature-dependent dielectric function of silicon comes from two physical factors: the thermal phonon population and the temperature variation of the band gap.

As an additional study, we examined the phonon assistance to NRET from QDs into silicon with increasing temperature, by performing TRF measurements at temperatures higher than room temperature. To this end, we prepared and employed a new sample without using an alumina spacer. For the experimental characterization, we worked in the range of the

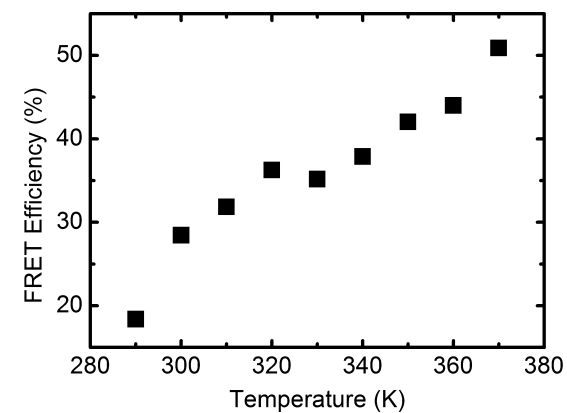

Figure 7. FRET efficiency as a function of temperature in the range $290-370 \mathrm{~K}$.

elevated temperatures possible in our time-resolved setup. Here we observed that the NRET process becomes more efficient going from 290 to $370 \mathrm{~K}$ as presented as a function of temperature in Figure 7. The observed increase in this temperature range is attributed to enhancement of the phonon-assisted interband transitions in silicon owing to occupation of the phonon modes in silicon even if the PL QY of the QDs is expected to be comparatively reduced due to the thermal droop. Therefore, the net observed result is that the phonon-assisted enhancement in interband transitions in silicon is strong enough and enhances NRET with increasing temperature in spite of various adverse effects there might be in the studied temperature range. This finding indicates the technological importance of the phonon assistance in the optical sensitization in silicon devices and may open up new possibilities for enhanced silicon photonics including photovoltaics and photodetectors.

\section{CONCLUSIONS}

In summary, we presented and analyzed the temperature-dependent fluorescence lifetimes and energy transfer efficiencies in hybrid QD-silicon nanostructures to reveal dynamics of the FRET based on the phonon assistance for the absorption process. To study systematically the underlying physics of FRET, we utilized the QD-silicon nanostructure platforms with varying separation thicknesses between the donor QDs and the acceptor silicon. Our experimental data showed that FRET from the QDs into silicon substrates becomes weaker at low temperatures, which is attributed to the phonon-involved interband transitions in silicon. To explain these experimental measurements, we first developed a temperature-independent analytical model using dipole-dipole interaction, leading to $\propto d^{-3}$ distance dependence and then suggested a modified physical model introducing temperature effects based on phonon-assisted absorption into this analytical model as a semiemprical approach. The model proves to be successful in analyzing and explaining temperature dependence, leading to reasonably well matching results with the experimental data. In addition, the full temperature-dependent model, which also takes temperature-dependent QY changes 
of the donor QDs into account, is presented to improve the match between the theory and the experiments. Therefore, these findings indicate the assistance of phonons in FRET for the case of indirect band-gap acceptors such as silicon as the phonon assists for the case of optical absorption.

\section{METHODS}

The average radius of the QDs was obtained by an FEI Tecnai G2 F30 ensemble HR-TEM. Film thicknesses of the native oxide and the QD layer on silicon substrates were recorded by a V-VASE ellipsometer. We deposited $\mathrm{Al}_{2} \mathrm{O}_{3}$ thin films on $\mathrm{SiO}_{2} / \mathrm{Si}$ substrates using $\mathrm{Al}_{2}\left(\mathrm{CH}_{3}\right)_{6}$ and $\mathrm{H}_{2} \mathrm{O}$ precursor gases at $200{ }^{\circ} \mathrm{C}$ with a Savannah 100 ALD system. The QDs were spin-coated over our $\mathrm{Al}_{2} \mathrm{O}_{3} / \mathrm{SiO}_{2} / \mathrm{Si}$ structures at $2000 \mathrm{rpm}$ for $150 \mathrm{~s}$. To confirm the uniformity of surface coverage for the QDs, the image of the QDs coated on the samples was taken by a Quanta 200 FEG Environmental SEM with high magnification. To assess the FRET dynamics in the QD-Si hybrid structures, fluorescence decays of the QDs were recorded by TRF spectroscopy with a closed cycle He cryostat.

For the temperature-dependent $\mathrm{OY}$ measurements of the $\mathrm{QDs}$, we recorded $\mathrm{PL}$ intensities for each temperature using TRF spectroscopy with a closed cycle He cryostat. We measured the counts at the wavelength range of $520-610 \mathrm{~nm}$ with $10 \mathrm{~nm}$ steps and summed them up to obtain the total photon counts at each temperature. We set the accumulation time at each wavelength as $20 \mathrm{~s}$ since this gives approximately the real time of the TRF lifetime measurements we presented in the study. As given in Figure S2 (Supporting Information), as the temperature decreases, the QY increases gradually, making a peak at around $177 \mathrm{~K}$, corresponding to the toluene freezing temperature, and then decreases slowly at lower temperatures. The solvent phase transition of the colloidal QDs at low temperatures may affect the temperature dependence behavior of the QDs' PL intensity. ${ }^{30}$ In addition, photo-oxidation and photobleaching may occur as a result of the long duration of illumination in the TRF measurements. ${ }^{32,33}$

Conflict of Interest: The authors declare no competing financial interest.

Supporting Information Available: Figures, text, and tables giving the model of phonon-assisted FRET from CdSe/ZnS QDs to monocrystalline bulk silicon, correction for the radiative lifetime of QDs due to the refractive index difference in the substrate, analysis of temperature-dependent luminescence lifetimes of the QDs, and analysis of interdot FRET between the QDs and theoretical parameters. This material is available free of charge via the Internet at http://pubs.acs.org.

Acknowledgment. This work is supported in part by EU-FP7 Nanophotonics4Energy NoE and TUBITAK EEEAG 109E002, 109E004, 110E010, and 110E217, and in part by NRF-CRP-62010-02 and NRF-RF-2009-09. H.V.D. acknowledges support from ESF-EURYI and TUBA-GEBIP, and A.Y. from TUBITAK BIDEB. A.O.G. was supported by the U.S. Army Research Office (USA) under grant number W911NF-12-1-0407. The authors acknowledge Kivanc Gungor for the SEM imaging. We are also pleased to acknowledge one of the anonymous reviewers for suggesting that we study NRET at temperatures higher than room temperature.

\section{REFERENCES AND NOTES}

1. Müller, A.; Ghosha, M.; Sonnenschein, R.; Woditsch, P. Silicon for Photovoltaic Applications. Mater. Sci. Eng., B 2006, 134, 257-262.

2. Stelzner, T.; Pietsch, M.; Andra, G.; Falk, F.; Ose, E.; Christiansen, S. Silicon Nanowire-Based Solar Cells. Nanotechnology 2008, 19, 295203.

3. Garnett, E. C.; Yang, P. D. Silicon Nanowire Radial P-N Junction Solar Cells. J. Am. Chem. Soc. 2008, 130, 92249225.
4. Yu, Z.; Raman, A.; Fan, S. Fundamental Limit of Light Trapping in Grating Structures. Opt. Express 2010, 10, 366-380.

5. Tian, B. Z.; Zheng, X. L.; Kempa, T. J.; Fang, Y.; Yu, N. F.; Yu, G. H.; Huang, J. L.; Lieber, C. M. Coaxial Silicon Nanowires as Solar Cells and Nanoelectronic Power Sources. Nature 2007, 449, 885-889.

6. Jang, M. S.; Atwater, H. Plasmonic Rainbow Trapping Structures for Light Localization and Spectrum Splitting. Phys. Rev. Lett. 2011, 107, 207401.

7. Mutlugun, E.; Soganci, I. M.; Demir, H. V. Photovoltaic Nanocrystal Scintillators Hybridized on Si Solar Cells for Enhanced Conversion Efficiency in UV. Opt. Express 2008, 16, 3537-3545.

8. Guzelturk, B.; Mutlugun, E.; Wang, X.; Pey, K. L.; Demir, H. V. Photovoltaic Nanopillar Radial Junction Diode Architecture Enhanced by Integrating Semiconductor Quantum Dot Nanocrystals as Light Harvesters. Appl. Phys. Lett. 2010, 97, 093111.

9. Trupke, T.; Green, M. A.; Wurfel, P. Improving Solar Cell Efficiencies by Down-Conversion of High-Energy Photons. J. Appl. Phys. 2002, 92, 1668-1674.

10. van Sark, W. G. J. H. M. Enhancement of Solar Cell Performance by Employing Planar Spectral Converters. Appl. Phys. Lett. 2005, 87, 151117.

11. Förster, T. Zwischenmolekulare Energiewanderung und Fluoreszenz. Ann. Phys. 1948, 437, 55-75.

12. Stavola, M.; Dexter, D. L.; Knox, R. S. Electron-Hole Pair Excitation in Semiconductors via Energy Transfer from an External Sensitizer. Phys. Rev. B 1985, 31, 2277-2289.

13. Alivisatos, A. P.; Arndt, M. F.; Efrima, S.; Waldeck, D. H.; Harris, C. B. Electronic Energy Transfer at Semiconductor Interfaces. I. Energy Transfer from Two-Dimensional Molecular Films to Si(111). J. Chem. Phys. 1987, 86, 6540.

14. Lu, S.; Madhukar, A. Nonradiative Resonant Excitation Transfer from Nanocrystal Quantum Dots to Adjacent Quantum Channels. Nano Lett. 2007, 7, 3443-3451.

15. Lu, S.; Lingley, Z.; Asano, T.; Harris, D.; Barwicz, T.; Guha, S.; Madhukar, A. Photocurrent Induced by Nonradiative Energy Transfer from Nanocrystal Quantum Dots to Adjacent Silicon Nanowire Conducting Channels: Toward a New Solar Cell Paradigm. Nano Lett. 2009, 9, 4548-4552.

16. Nguyen, H. M.; Seitz, O.; Aureau, D.; Sra, A.; Nijem, N.; Gartstein, Y. N.; Chabal, Y. J.; Malko, A. V. Spectroscopic Evidence for Nonradiative Energy Transfer between Colloidal Cdse/Zns Nanocrystals and Functionalized Silicon Substrates. Appl. Phys. Lett. 2011, 98, 161904.

17. Nguyen, H. M.; Seitz, O.; Peng, W.; Gartstein, Y. N.; Chabal, Y. J.; Malko, A. J. Efficient Radiative and Nonradiative Energy Transfer from Proximal CdSe/ZnS Nanocrystals into Silicon Nanomembranes. ACS Nano 2012, 6, 55745582.

18. Labeau, O.; Tamarat, P.; Lounis, B. Temperature Dependence of the Luminescence Lifetime of Single CdSe-ZnS Quantum Dots. Phys. Rev. Lett. 2003, 90, 257404.

19. Chance, R. R.; Prock, A.; Silbey, R. Advances in Chemical Physics. In Molecular Fluorescence and Energy Transfer Near Interfaces; Prigogine, I., Rice, S. A., Eds.; Wiley: New York, 1978; pp 1-65.

20. Novotny, L.; Hecht, B. Principles of Nano-Optics. In Dipole Emission Near Planar Interfaces; Cambridge University Press: Cambridge, 2007; pp 335-346.

21. Whitmore, P. M.; Alivisatos, A. P.; Harris, C. B. Distance Dependence of Electronic Energy Transfer to Semiconductor Surfaces: $3 n \pi *$ Pyrazine/GaAs(110). Phys. Rev. Lett. 1983, 50, 1092-1094. 
22. Platzman, P. M.; Wolf, P. A. Waves and Interactions in Solid State Plasma; Academic Press: New York, 1973.

23. Govorov, A. O.; J. Lee, J.; Kotov, N. A. Theory of PlasmonEnhanced Förster Energy Transfer in Optically Excited Semiconductor and Metal Nanoparticles. Phys. Rev. B 2007, 76, 125308.

24. Hernandez-Martinez, P.; Govorov, A. O. Exciton Energy Transfer between Nanoparticles and Nanowires. Phys. Rev. B 2008, 78, 035314.

25. Palik, E. D. Handbook of Optical Constant of Solid; Academic Press: New York, 1985.

26. Kagan, C. R.; Murray, C. B.; Bawendi, M. G. Long-Range Resonance Transfer of Electronic Excitations in ClosePacked CdSe Quantum-Dot Solids. Phys. Rev. B 1996, 54, 8633.

27. Lunz, M.; Bradley, A. L.; Chen, W. Y.; Gun'ko, Y. K. TwoDimensional Forster Resonant Energy Transfer in a Mixed Quantum Dot Monolayer: Experiment and Theory. J. Phys. Chem. C 2009, 113, 3084.

28. Bucher, K.; Bruns, J.; Wagemann, H. G. Absorption Coefficient of Silicon: An Assessment of Measurements and the Simulation of Temperature Variation. J. Appl. Phys. 1994, 75, 1127-1132.

29. Yu, P.; Cardona, M. Fundamentals of Semiconductors. Physics and Materials Properties, 4th ed.; Springer: Berlin, 2010.

30. Lee, J.; Govorov, A. O.; Kotov, N. A. Bioconjugated Superstructures of CdTe Nanowires and Nanoparticles: Multistep Cascade Forster Resonance Energy Transfer and Energy Channeling. Nano Lett. 2005, 5, 2063-2069.

31. Antipov, A.; Bell, M.; Yasar, M.; Mitin, V.; Scharmach, W.; Swihart, M.; Verevkin, A.; Sergeev, A. Luminescence of Colloidal CdSe/ZnS Nanoparticles: High Sensitivity to Solvent Phase Transitions. Nanoscale Res. Lett. 2011, 6, 142

32. Van Sark, W. G. J. H. M.; Frederix, P. L. T. M.; Van den Heuvel, D. J.; Gerritsen, H. C. Photooxidation and Photobleaching of Single CdSe/ZnS Quantum Dots Probed by RoomTemperature Time-Resolved Spectroscopy. J. Phys. Chem. B 2001, 105, 8281-8284.

33. Manner, V. W.; Koposov, A. Y.; Szymanski, P.; Klimov, V. I.; Sykora, M. Role of Solvent - Oxygen Ion Pairs in Photooxidation of CdSe Nanocrystal Quantum Dots. ACS Nano 2012, 6, 2371-2377. 